\title{
ACTION, INTERACTION AND THE ROLE OF AMBIGUITY IN THE INTRODUCTION OF MOBILE INFORMATION SYSTEMS IN A UK POLICE FORCE
}

\author{
Dr David Allen and Professor T. D Wilson \\ Leeds University Business School, The University of Leeds, Leeds, LS2 $9 J T$
}

\begin{abstract}
Mobile wireless computing has been identified as a critical new application of information technology; however, only a few case studies are available focusing on the organisational or social issues related to the deployment of these technologies. This paper provides a rich description of the situated activities of a particular set of mobile workers (police officers) during the implementation of mobile wireless laptops. The paper describes two implementations of one type of technology in a single organisation. The implementations took very different trajectories; in one the technology was resisted and eventually withdrawn while in the other it was embraced and embedded into working practices. The paper clearly demonstrates how ambiguity, over time, influenced the trajectory of the implementation process in the different sites. The paper explores the use of an alternative theoretical approach to the use of technological frames to understand ambiguity and implementation of technology: Strauss's Theory of Action. Using Strauss's concept of trajectory the paper demonstrates the importance of understanding the relationship between ambiguity and the implementation of mobile information technology.
\end{abstract}

Key words: Mobile Information Systems, Police, Ambiguity

\section{INTRODUCTION}

Mobile computing has been heralded as the next major paradigm in personal computing (Okoli, Ives et al. 2002). It is unsurprising, therefore, that over the last two years there has been a rapid growth in the literature and 
research relating to the technical and commercial aspects of the application of mobile devices (Siau, Ee-Peng et al. 2001). A number of researchers, however, have indicated that there are 'few studies of the use of these mobile devices' Wilberg (2001)). (Kakihara and Sørensen 2002) have pointed to the pressing need for further theoretical and empirical work in this area. In particular, there is a need for IS research to be actively involved in studies where technologies are being built and tried out - before they reach the marketplace (Lyytinen and Yoo 2002). This paper provides one such empirical study approaching the research from an alternative perspective: mobile informatics (Dahlbom and Ljungberg 1998; Kakihara and Sørensen 2002; Nulden and Lundin 2002), or analysing the use of mobile devices in a particular setting.

The paper analyses the implementation of a mobile office solution in two sites in a large UK police force (Surrey Police Force). This presents an intriguing case: one where the implementations in the two sites took very different trajectories; in one the technology was resisted and eventually withdrawn while in the other it was embraced and embedded into working practices. As the research progressed it became clear that the role of ambiguity during the process of implementation was particularly significant. In attempting to understand this, the researcher turned to the body of work about how people make sense of ambiguity (multiple meanings) created by the implementation of IT. There is only a small amount of literature which focuses on the collective creation and shaping of interpretations for understanding and enacting IT (Henfridsson 2000). Much of this work draws upon sense-making perspectives and also utilizes a cognitive perspective.

One of the most influential works in this area is that of Orlikowsi and Gash (1994) who introduced the idea of technological frames. They described these as schema used by actors to interpret technology, arguing that when there is ambiguity or difference between individuals' technology frames the implementation will be more problematic. Henfridsson (2000), however, argues that this work places too much emphasis on the reduction of ambiguity between different stakeholders' assumptions, beliefs and values about a technology. He points out that the work of Ciborra and Lanzara (1994) indicates the importance of ambiguity in stimulating innovation and learning and he puts forward an alternative approach, suggesting that ambiguity can be managed most effectively when there is a transition between states of ambiguity about the use and role of the technology and states of common sense where there is no ambiguity. Indeed, he argues that ambiguity should be created around technologies in order to make the most of IT adoption. In contrast, Davidson's (2002) research, from a quantitative and positivist perspective, reinforces and develops Orlikowski and Gash's use of technological frames, and identifies four frame domains that are 
pertinent during the information systems development (ISD) process. She indicates that repeated shifts in salience between two specific technological frames (related to the business value of IT and IT delivery) can disrupt the information systems development process. In common with the earlier work of Orlikowski and Gash, Davidson seems to argue that, by recognizing (and therefore reducing) ambiguity, the process will be improved: “...if shifts in frame salience are recognized and explicitly acknowledged, ISD participants may be better able to manage" (Davidson 2002:353). Barrett (1999) develops Orlikowski and Gash's work in another direction augmenting their socio-cognitive approach with 'structural culture' (Thompson 1990; Thompson 1995) from a critical perspective. Barrett argues that a key reason for low levels of EDI adoption is an incongruence of cultural assumptions among different sub-cultures. Mantovani and Spagnolli, (2001), working from within Actor Network Theory, add another dimension to the debate by indicating that the existence of ambiguity about norms and technology is particularly useful at the start of the implementation of a technology, because it allows the expression of different interests of actors involved in the implementation. They also argue that the ambiguity associated with norms and technology will naturally be reduced during the process of implementation as 'shared lines of interpretation crystallise.' (Mantovani and Spagnolli 2001:317)

While the findings of these approaches are useful and relevant, it could also be argued that they place too much emphasis on socio-cognitive structures determining and shaping action and too little emphasis on the way socio-cognitive structures are contested and shaped over time. As Ciborra stated, in understanding IT implementation there is a need to bring back into the picture the situation of the actor (Ciborra 1999). While this seems to be acknowledged in IS research, it is achieved, mainly, by the introduction and adaptation of ideas from cognitive psychology (c.f. Tan and Hunter 2002). While providing a useful and interesting contribution, the application of socio-cognitive theory can be criticized on a number of grounds:

- It fails to acknowledge the emotional aspect of organizing (Fineman 1996; Ciborra 1999).

- The cognitive view has only recently achieved widespread acceptance in organizational studies (Hodgkinson and Sparrow 2002) and, therefore, the language of cognition is still diffuse and conflicting. Walsh (1995) identified more than 90 terms related to cognition in the language of management theory. Translation from its use in organizational studies to information systems or information management should be undertaken cautiously.

- Cognitive theory has developed in a behaviourist tradition (positivistic and nomethetic), but it is being used in an interpretive (ideographic) 
tradition in the field of information systems (Orlikowski and Gash 1994). Some researchers point to this use in ideographic frameworks as positive, but it has been criticised in the mainstream management literature. Tan and Hunter (2002), for example, cite the work of Daniels, Johnson, and de Chernatony (1994) as a useful route for IS researchers to follow, but this work has been roundly criticized by mainstream cognitive researchers in management (Hodgkinson 2002) as theoretically confused.

- Socio-cognitive approaches are used from an interpretative perspective, but are operationalised in a way that suggests that cognitive structures are impermeable and create action; whereas we argue that structures are constantly created and recreated in action.

The first section of the paper presents a brief background and context to the case study. The second section presents the theoretical position and methodology. The next section presents a description of the initial sensemaking about the introduction of the technology in two sites, analysing them through the lens of the temporal and spatial aspects of mobile work. It also provides an analysis of the relationship between new information behaviour enabled by the technology and power relations. This section identifies the initial commonality of opinion and a common trajectory of the implementations in the both sites. The fourth section goes on to describe the way sense-making about the implementation and the trajectories of the implementation diverged. The paper concludes with a discussion of the results and their implications for research and practice.

\subsection{Background to the Implementation}

Police forces in the UK are at radically different stages of development in their use of information systems (O'Dowd 1998; Povey 2001). With strong support from the UK Government they are now placing more emphasis upon the use of technology to support all aspects of their work. The intention is for many aspects of their operations to be transformed by 2005. This has been labeled 'e-policing' (Povey 2001), a key aspect of which is mobilising information (Povey 2001) to make it available to officers wherever they are working through the use of mobile phones, mobile data terminals and mobile computing devices (wireless laptops and Personal Data Assistants (PDAs)). One of the leading forces in the UK in the deployment of mobile systems is Surrey Police, which is based in the south of England, sharing a boundary with London. Its area of operation includes urban and rural areas and involves the management of a number of varied policing environments, from Heathrow Airport to rural villages.

This paper discusses the implementation of a specific mobile technology: the Surrey Police Remote Officer and Vehicle EnviRonment (ROVER) 
units. ROVER is essentially a laptop computer with a GSM mobile phone modem allowing officers in any location to access the Force intranet, the Surrey Police Information and Knowledge Environment (SPIKE). This gives officers access to police-specific software (such as national databases of legal information or information on holders of firearms) and generic software (e-mail or word processing software) from a laptop with wireless access to the organisation's intranet. It is important to note that this police force had mobilised its own network environment; thus, the officers were not faced with a new interface or new software. Two implementations were studied: the first was in a small town with uniformed patrol officers, the second was a larger town with plain-clothes detectives.

\section{THEORETICAL POSITION AND METHODOLOGY}

At the start of the project (October 2000) very little research on the use of mobile wireless technologies was available and no empirical research was located on the organisational aspects of their use in the police. The objective of the research, therefore, was to explore the situated activities of these mobile workers, to produce a rich description of the implementations.

While aware of the research on adoption and assimiliation of information systems (c.f., Gallivan 2001), the primary approach in the IS literature has been through the use of the Technology Acceptance Model (TAM) (Adams, Nelson et al. 1992; Agarwal 2000; Venkatesh 2000). In this research we wished to take a different approach. Much of the TAM literature focuses on the individual level and is nomothetic and positivistic. In contrast this research project focused on the group level and works from within an interpretive framework: drawing on interactionism, pragmatism and utilizing ideographic tools.

In an attempt to place more emphasis on the interaction between structure and action over time, and to illuminate the contested and changing nature of structures, Strauss's (1993) Theory of Action is used here. In particular, four concepts are drawn upon: trajectory, trajectory phasing, trajectory projection, and orders. Trajectory refers both to the course of any observed phenomenon as it evolves over time and the actions and interactions contributing to its evolution. Strauss uses the term to encompass both a predetermined course of action and interaction in ways that are unanticipated and not entirely manageable. He argues that researchers and those involved in the interaction can distinguish phases in the trajectory of a phenomenon. He illustrates this point by drawing on his earlier work (Glaser and Strauss 1968) where the phase of 'they are dying' was identified by those involved 
in the trajectory of terminal illness. The term technological trajectory has been used in a similar way by Jenkins and Floyd (2001). The third concept used in this paper is that of trajectory projection. This refers to a vision of the expected course of interaction, which may or may not be shared by the actors. Strauss also identifies "orders", defined as analytic abstractions that summarize what the actions and interactions involved in a phenomenon are intended to achieve. The orders used here are: temporal order, spatial order, work order, informational order and technological order. The term "temporal order" (Strauss 1993) is used to refer to the frequency, scheduling, pacing, duration and timing of actions. The term "spatial order" refers to "how objects are arrayed in given spaces; how actions take place or are supposed to take place in certain spaces; the symbolism associated with various spaces" (Strauss 1993: 59) "Work order" refers to the way in which work is organised. Informational order refers to the flow of information between actors. The final order, "technological order", refers to the organisation of technological artefacts and the routines that surround them

We hold that the Theory of Action and the concepts of 'trajectory' and 'orders' are particularly useful in research on mobile data applications. The point must be stressed that the term 'mobile data' is not entirely adequate, since in the police applications, it is the person who is mobile, while, especially in the case of thin-client systems, the data remain on the server until pulled by or pushed to the user. The temporal order is critical in police work where the rapid availability of information in a stop-and-search event may make the difference between a successful arrest and a need to let the suspect go free, or where the scheduling of actions in response to an incident may make the difference between the effective and ineffective use of people. The spatial order is also highly relevant, as the police officers are continually on the move from one location to another either on foot or in police vehicles and some of the spaces they occupy have greater or lesser degrees of symbolism associated with them. A suspect on the street, a public place, is subject to less pressure than the same person in a police car, or removed to a cell in a police station. From a police perspective, the more sure and the more rapid the transfer from one to another can be made, the better.

The discussion is based on qualitative data from the study of the implementation of ROVER over a twelve-month period. Although data gathering techniques included the analysis of project documentation and observation, the primary method used was semi-structured interviews. Respondents included senior police officers in Police Headquarters (who had approved the implementation), senior police officers involved at a Divisional Level in the implementation (and who had requested the implementation), IT Services (including the IT Support Officer, those involved in offering training on the system, the Knowledge Manager involved in the process of 
implementation and the Director of IT Services). Individual interviews were also conducted with the senior staff in both police stations (Inspectors and Sergeants) and most officers involved in using the technology. The more junior police officers were interviewed either in focus-group and/or individual interviews.

Observation of working practices and information behaviour also took place, but only in the Police Stations. This was a condition of gaining entry for the research. A limitation on the findings was that observation of use of the technology 'in the field' could not be undertaken. This issue is being addressed in further studies in other police forces.

Karahanna, Straub, and Chervany (1999) note that few research projects have dealt with pre-adoption and post-adoption beliefs and attitudes in IT implementation. The data collection for this research project was undertaken in three distinct stages. The first stage was before the implementation, where attitudes and expectations of the implementation were discussed. The second stage was two months after implementation, to gauge initial reactions and to understand how the technology was being used and had influenced working practices. Finally, the last data collection visit took place twelve months after the implementation to understand how the technology was shaped during the implementation and how the innovation had shaped organisational practices. The case study presented below is the authors' interpretation of the data collected through this process.

\section{IMPLEMENTATION: INTIAL STAGE OF THE TRAJECTORY}

The initial stage of the trajectory was characterised by a lack of ambiguity over the implementation of the technology. The impetus for the initiation of the ROVER came not from the Information Management or Information Technology Service in the police force, but from the users and, in particular, Senior Operational Officers in the Uniformed Division. When interviewed, these senior officers had a clear vision for the future trajectory of the implementation: it would increase operational efficiencies by changing the working practices of officers; in particular it would influence spatial and work orders. They described this through the metaphor of reducing the "yo-yo effect": a process by which police officers had to return numerous times during the day to the police station to communicate or process information before returning to the field. It was believed that, if the number of times the officer had to return was reduced, officers would 'stay out on the street' for longer periods and so increase the visibility of uniformed police (an important objective set for them by Central 
Government). They also wanted to shape informational orders: there was a hope that the ROVER would enable a more effective approach to policing by providing information when and where police officers need it; thereby supporting an intelligence-led, pro-active style of policing.

The initial implementation in both sites, was perceived in a positive manner by the officers involved. Officers gave accounts that indicated that they felt more informed, made better use of existing internal information resources and had located new external sources. They also felt that their external communication had improved and, critically, they felt that the use of the ROVER helped create a perception in the public that a higher quality of service was being delivered. The two implementations of the ROVER seemed to take similar trajectories: at both sites it was initially reported as extremely successful, gaining support from senior managers, uniformed officers and plain clothes detective officers. The way the technology was perceived to have shaped behaviour initially can be seen through the accounts illustrating three key elements which changed after the implementation: the changes in temporal, spatial, information, and work orders.

\subsection{Temporal Order}

A number of researchers have argued that the 'network society' is characterised by the decline of any rhythm or cycle of life, either biological or social (Castells 1996). While the relationship between time and technology is a contested one (Frissen 2000), information systems researchers have presented a sophisticated perspective of the influence of technology on time (Lee 1999; Lee and Liebenau 2000; Orlikowski and Yates 2002), particularly in the area of IS development (Nandhakumar 2001). In both implementation sites accounts that identified the impact of the implementation on the temporal order of actions were identified. One example of this is the way uniformed officers reported that they could change the way they input and updated crime reports on the crime information system (CIS). They could, for example, input information while it was 'fresh in their minds' whilst they were on patrol. A probationary, uniformed officer noted that, using the traditional approach, he could accumulate three or four reports in his notebook as he was sent from incident to incident. It could then be perhaps 24 hours later before he was able to update the system. Using ROVER, information could be input into CIS in 'real time' and it was said that information entered would not only be more 'up-to-date', but would also be more comprehensive and accurate. Another PC stated: 
"I use it quite a lot - linking up when I am a passenger in a car looking at current jobs and my workload, doing checks on vehicles rather than doing it over the air, updating crime reports at the scene. It is very useful for that, I can go in, do the update while I am standing there talking to them and if they ask any questions I have got it at my fingertips. I don't have to do it from memory and I can give them the relevant numbers. "(Uniformed PC)"

Similarly, CID Officers described the way they were using the technology to overcome temporal and spatial issues. One officer noted the way it enabled him to become better informed and more focused when called out to an incident, allowing him to access the force system into which crimes were stored after being reported by the public (the ICAD):

"The only time I take it home is when I'm on call. The advantages are at 3 o'clock in the morning you get a phone call, the control room only through necessity only ever give us three lines: 'It's such and such an incident, there's a bloke in custody and it happened in .....' 'Oh great, what about this?' 'Don't know!' You are talking to people third hand. So what you can do ... look at the actual ICAD as well as get a feel for the job before you actually speak to anyone."

In the initial phase of the implementation, account of the use of the technology to overcome temporal barriers was common in both implementation sites, and was supportive of the implementation.

\subsection{Spatial Order}

The spatial concept of location is one which, as Healey and Reeves (2001) point out, is the most intuitive framework for understanding mobile work. The role of information technology in making it possible for spatially or organizationally distant parties to communicate has been a recurring theme in much of the literature on CSCW (DeSanctis and Monge 1999). It is unsurprising, therefore, that recent work on mobile technologies and mobile work practices identifies it as a key dimension of mobile work (Kakihara and Sørensen 2001; Kakihara and Sørensen 2002).

Much of the language that legitimised the implementation of the ROVER emphasised the spatial order of action. Officers described the way they could use the ROVER to be 'informed' at remote locations and, therefore, work faster and more effectively. One officer described how he used the system in the Police office in the Crown Court. He was able to use ROVER to find information to resolve questions put to him and thereby 'appear more professional'. He stated: 
"They were asking me questions, the defence were coming up with items and the prosecution were saying that they wanted this, this, and this. And instead of my rushing off for hours and trying to find someone to ask, I would just turn this on and answer it within a couple of minutes...it speeded the whole process up."

Most of the officers interviewed noted the importance of the mobile data link, stating that they were often working in environments (often outside the area covered by the police force) where they were unable to get access to a landline. A number of the officers told how they could use the ROVER in locations where radio contact was difficult.

Accounts about the use of the technology to overcome spatial barriers seemed to permeate early discourses within both sites, reinforcing the perception of a rapid internalisation, use and acceptance of the technology within the organisation.

\subsection{Information Order}

The use of the wireless laptops augmented existing information channels (e.g., police radio) and allowed police officers direct access to remote databases. This removed existing information intermediaries (such as control room operators) who had provided the only form of access to information held in police databases via the police radio. The control room operator plays a key role in most police forces; they take calls from the public, enter the information into police information systems and call officers on the radio in order to dispatch them to a respond to calls. They also play a pivotal role in the information gathering by the police officers as they provide the only remote access to police information systems. The police officers would use a shared radio system to contact the control room operators, therefore, conversations could be overheard by other officers (peers and superiors). The use of the ROVER units, therefore, moved the information behaviour of officers in the field from a position where information seeking and use was observed, shared and reliant up a third person to a position where it was closed to observation and individual. Officers, particularly younger officers who feared looking foolish in the eyes of their team, or censure from their sergeant for asking too many questions or taking up too much time on the radio said that they were more likely to search for information directly using the ROVER units. The relationship between control operators and patrol officers can sometimes be strained because of their differing reward systems and objectives. The control operators often have competing demands on their time and are evaluated on the speed with which they deal with a call. The patrol officers, on the other hand, require information with a high degree 
of accuracy and depth. The use of the ROVER units to bypass the control operators was seen as particularly useful, and was reported to improve the effectiveness and safety of officers. It seemed that officers were much more likely to search for information on the mobile terminal than by using traditional channels, precisely because it was not observed: officers' information seeking was not exposed to the comment and scrutiny of senior officers. It was also clear that they became more active information gatherers; rather than waiting to be informed, they used the technology to scan their environment and look for information, or reports to which they could add their information. This was particularly true whilst officers were working on nights or during quiet periods on patrol. One young officer said he used the technology to access the Police National Legal Database (a repository of information about police legal powers):

"I used PNLD when I was out. It gets a bit embarrassing when you don't know the law. If you have got it to hand, before you pop in the house to speak to them you can just think look it up on PNLD and I will be up to speed, you are more aware of your power to deal with things."

This was particularly important as it reinforced the police officer's core values: the need to be seen by the public to be informed and enabling officers to understand the limits of their own powers (such as the power of arrest).

\section{IMPLEMENTATION: DIVERGENCE AND CRYSTALISATION OF TRAJECTORIES OF INTERPRETATION}

The initial reactions to the implementation were similar, but, as the implementation continued the trajectories diverged. By the end of the project many (if not most) of the uniformed officers had stopped using the technology. One officer noted:

"It made my life easier not to get the laptop out of the bag".

This change in attitude was particularly puzzling because at the start of the project uniformed officers were all very supportive of the technology. One officer noted:

"People were initially very keen, very into it, changing their desk tops and got it all sorted. It was a neat bit of kit." 
The resistance was such that the project with the uniformed officers was abandoned. However, the detective officers remained enthusiastic, actively using the technology. One detective commented in an interview that: "It would have to be a very big bloke that comes and takes my computer off me." Another stated that she though the technology was "... fantastic and I would now hate to be without it, I've quickly got used to the flexibility of it." The following text analyses the sense-making as conflicting interpretations of the technology developed, were contested and began to converge upon a dominant perspective and "crystallize in shared lines of interpretation" (Mantovani and Spagnolli 2001:317) this dominant perspective was represented in the renegotiation and interpretation of the perceived impact of the technology.

\subsection{The Implementation in the Uniformed site}

The implementation in the uniformed police station was characterised by a high degree of ambiguity about acceptable use of the technology, ambiguity about the permanence of the implementation, and ambiguity about the reasons for the implementation. Ambiguity was present because different interpretations were supported at the same time (Weick 1995). Without objective criteria given for the implementation the officers relied upon professional values to make sense of the situation, using these values to extrapolate from the data available. It is significant that the officers read from the facts according to their professional value system, divergence in sense-making being linked to the existence of different sets of values. This will be discussed further in the next section of the paper. As the implementation progressed the technology was increasingly perceived as threatening a particular set of officers' values. The role and nature of the implementation became highly ambiguous. McCaskey (1982) identifies characteristics of ambiguity as: multiple, conflicting interpretations, different value orientations and political and emotional clashes. The Uinformed site became one in which discourses about ambiguity and anxiety (Baruch and Lambert 2002) rapidly dominated.

\subsubsection{Work Order and ambiguity;}

This ambiguity was demonstrated by one officer in his discussion of his use of the technology. As already noted, the system allowed officers to use the technology to change their information behaviour and their work practices. For example, one officer noted that use of the technology allowed him to park his car and access his e-mail from wherever he was: 
"Promoted my pulling up and using it to see, pulling up on nights and saying to myself 'is there anything about' and using the terminal - quickly check my mails to see what was going on at 2.00am in the morning.'

While this sort of behaviour was seen as beneficial to the officer and his team, there was still a great deal of ambiguity about the acceptability of these behaviours. The same officer, for example, went on to say that he was concerned what the reaction of his senior officers would be to his remote use of the technology.

"I felt that if a governor pulled up next to me, I would think - what do I say? I am checking my e-mails, using CIS, PNLD etc [I was] worried that I would be seen as a bit of a skiver [i.e., avoiding work]".

The ambiguity about the new behaviours was compounded by ambiguity over the permanence of the implementation. Organisational history is important in that the contextual conditions and work itself has a history (Strauss 1993:89). History can become embodied within work, in routines and norms, and thereby be rendered invisible. For example, as work evolves over time, routines, behaviour and attitudes are woven into work order and remain, often after their origins are forgotten. These routines are 'blackboxed' and are only questioned when the work order is challenged and sense-making Weick (1995) occurs. During sense-making the past is interpreted and reinterpreted by actors in order to make sense of change in the work order. When the technology is implemented it begins to challenge embedded routines, as the technology enables the actors to evolve new routines and behaviour with the technology. As the implementation progressed, confidence in the implementation was affected as officers drew on accounts of the past implementations to understand the current implementation.

This police force has a particularly proactive and innovative Information Technology Service which is at the vanguard of changes to the use of information technology in the police service and is recognised as one of the most technologically advanced in the UK. It has a history of testing and developing technologies in the field. Officers drew on their understanding of the past implementations of technology when making sense of the ROVER units. Crucially, operational staff saw the earlier use of laptops as unsuccessful. Although the case reported in this paper was the first deployment of wireless laptops the force had attempted a limited deployment of laptops on other occasions, one PC noted: 
"I have had two laptops before this one in the last year and they have been absolutely useless"

This particular station seems to have the status of a 'test bed' for some of this organisational and technological change. While this has positive implications because the staff took pride in this reputation, on the other hand many of these changes seemed to have been ephemeral in nature. The project was seen as just another experiment. The experimental status was also reinforced by the IT service who, recognising the relatively low cost of implementation and the fact that they were the first police force (and indeed one of the first organisations UK wide) to mobilise their whole intranet in this way, used the term 'experiment' to reduce risk, to involve the officers, and, therefore, to reduce resistance to the technology. This had the effect of increasing ambiguity about the implementation: in many ways it made it easy and acceptable for officers to stop using the technology.

As the implementation proceeded this ambiguity was compounded by an increasing concern about why the technology was being implemented. The uniformed officers felt that the operational future of their station (and therefore of their team) was in question. Officers interpreted the messages that they were given about the implementation by their senior officers as indicating that the successful implementation of the technology would lead to their Police Station being closed. One officer noted:

"I was sure that they also meant it to assist us with our job as well...we weren't sold on this. The American thing was sold a lot, that you can have your RV [meal break] outside, almost that you could almost not use the police stations anymore."

The American model of policing was explained as being one in which officers left the station in the morning and only returned at the end of their shift. To facilitate this, officers could take meal breaks outside the station in named restaurants. This would be a significant change in culture for officers in the force who, up to this point, were not allowed to eat in a public restaurant while wearing a uniform. This fear of dramatic changes to working practices and insecurity about the future of the police station created a general sense of insecurity, which limited innovative use of the technology.

Furthermore, the technology was interpreted as threatening the core values of the police officers: attacking their existing constructions of identity. The station culture in the uniformed patrol officers could almost be described as one of a 'siege mentality'. The impression gained was that the station was extremely hierarchical, and that the PCs were heavily reliant upon each other and their senior staff for guidance and support. There was a 
clear sense that their world-view was very much 'us against the world'. This was put to us as, 'if you offend one of us you offend all of us'. The impression was conveyed that the management style in action (as opposed the espoused management style) was a 'traditional' command and control approach based on 'rank determining role' and the restriction of the questioning of orders. This had a significant impact on the way officers were encouraged to use the system, as there was a mismatch between the espoused culture (the vision for policing offered by the Headquarters) and the culture in action as demonstrated in the station. One officer noted,

"In training I was told that the prevention was the most important part of side of the job; if you prevent it you will not have to respond to it. But it hasn't been happening like that because we have been busy and we haven't got the manpower.... We get tasked by three different sources... and they ask too much... [we] end up doing the reactive rather then the investigative, pre-crime work...You really don't get time."

Officers, initially, seemed to project their own expectations on to the technology. For some, ROVER was seen as a mechanism by which they could return to 'traditional' ways of policing. One officer, for example, noted that the implementation of technologies in the police force had led to them 'losing focus' and that this technology should help them get back to 'how it was and how it should be'. However, as the implementation progressed it became clear that the implementation of ROVER, rather than facilitating a return to the past, actually enabled new forms of work. This resulted in some officers feeling threatened by the technology. ROVER provided access direct access to the Surrey Police Information and Knowledge Environment (SPIKE), this is a rich information environment supportive of reflective and preventative police work. Although ROVER allowed access to information needed in responsive modes (what was referred to as fast- time) or use within conflict situations, it was not ergonomically designed to function effectively in these environments. While ROVER supported the mission of the force as a whole was not explicitly designed to support the values of the patrol officers. Indeed, it was increasingly seen as deeply threatening to the values and working practices of the uniformed officers.

\subsubsection{Spatial Order and ambiguity;}

The development of a strong team ethos based on face-to-face, peer-topeer information sharing was highly valued. One officer noted, 
"We are all very young in service, so we need people who have more experience so that we can sit and socialise with them and ask how would you deal with this...if you can sit face to face you can explain the thing more effectively".

The officers, therefore, placed a great deal of importance on co-location. This value was seen as being directly threatened by the implementation of ROVER. The senior officers in the station seemed to take a particularly close role in mentoring and managing the tasks undertaken by PCs. The inspector, for example, stated that she spent more time in the Constable's Report Room than in her own office. Senior officers stressed the importance of this face-to-face interaction in training their staff, but the author also gained the impression that there was a strong sense that this was also a mechanism by which they could control the behaviour of subordinates. Indeed, fear of loss of control was a key factor that influenced some of the officers' reaction to the technology. Officers expressed the concern that by using the technology they would have less control over their subordinates:

"There was fear that you would be out there on your own (because we are single crewed) for eight hours. I wouldn't be able to supervise people, lot of probationers, I wouldn't be able to go out with them and see what they are doing, because I would never see them. They used to come back regularly and I would say what are you doing now and someone would say, 'I have just been to this job and this happened'. So I would know what was going on. That wouldn't happen with the new system."

The issues of loss of control were also raised during the process of implementation. Initially, the implementation of ROVER was requested by the senior officer responsible for the station; however, as the implementation progressed senior officers in the station became increasingly concerned that they were losing control over the process of implementation and the ways in which the technology was being used. This was expressed through concerns about the communication of fault reporting and ideas about the use of the technology.

During the implementation and initial use of the technology sensemaking was (as Weick (1995) argues sense-making inevitably is): driven by as plausibility rather than accuracy. The implementation became a battle ground of values, the discourses which finally dominated being those which were most socially acceptable and credible within the group. Although this particular implementation was abandoned, users in other police stations have requested access to the technology and alternative modes of delivery of the mobile information to uniformed officers are being explored. The police 
station which had been the site of the implementation was closed and the officers redeployed elsewhere.

\subsection{The Plain Clothes Detectives}

In contrast to the unformed case, the implementation in the Detective Branch reduced ambiguity about the intention and use of the technology. The technology was also rapidly linked to the detective's values. These officers valued their independence and ability for autonomous decisionmaking: these were seen as being supported by the implementation of ROVER. In particular, the ROVER units were utilised in a way which supported the detectives spatial, temporal and work order. One of the ways in which this manifested itself was through descriptions of how ROVER had influenced the temporal and spatial order of the mechanism by which officers were able to make decisions on how to respond to a call out. A 'callout' was a situation where they would be asked to move to an incident and manage it (at any time of day or night). One officer stated that, in the past, they had to respond to all call-outs, often not being needed when they responded. By using ROVER they could often resolve the issues from home:

"It has given me a lot more flexibility in when I attend the Police Station or should I be called out. Do I actually have to leave my home or can I deal with it from home?"

Most officers noted that they worked over and above the normal shift hours. However, by using the ROVER they could perform any extra work as and when they wished:

"I do work 8 hours everyday, but it means that I can choose when I do extra and where I do the extra. It means I can see my daughter before she goes to bed and do a little bit afterwards if required. That's the usage I get from it."

The implementation of the technology did not threaten to change their existing work order with regard to social face-to-face interaction; rather it was seen as augmenting their existing working patterns, adding a further dimension rather than fundamentally changing working patterns. Thus, they saw ROVER as enabling greater communication between officers working on the same case, but working on different shift patterns. The temporal aspect of the implementation was one which was stressed by most of the officers interviewed, the way the technology influenced the traditional rhythms of police work was supportive of making individual police officers visibly more effective. One officer, for example, described how, when 
working on a rape case he was able to search on the Internet for information on drug rape.

The interpretations about the technology seemed to crystallize around these positive accounts. As ambiguity was reduced officers seemed more ready to invest in peer-to-peer support. A significant element of the learning that took place during the implementation, for example, seemed to be based on peer-to-peer support. Officers described this as spreading expertise. Activities like:

“...Doing mail merge for another officer, showing how to use the spreadsheet database for a stolen property enquiry."

This learning seemed to be taking place on a one-to-one basis, based on example. Officers who had taken on the units early in the implementation played an active role in this. The telling of stories of successful or unsuccessful use of ROVER was the main mechanism by which individuals' located people with relevant experience.

'If we are trained and know what to do, the uses we find will be conveyed by word of mouth.'

The computer services also managed the implementation process in the CID site slightly differently; in particular they placed heavy emphasis on factors which would reduce anxiety and ambiguity. For example, they identified a single 'enthusiast' for the technology and gave her a ROVER unit for three months before roll-out. This officer acted as an advocate for the roll-out and as an unofficial, first line help-desk when the ROVER units had been deployed. They also reviewed her use of the technology and supported her dissemination of 'best practice' in the use of ROVER to her team. They also based an IT support technician in the CID office in order to provide a rapid response to technical problems with the ROVER units and to and help users if they encountered difficulties.

\section{CONCLUSION}

Both these implementation sites characterise situations where individuals in a community attempt to make sense of the implementation of a technology which was (on first view) relatively simple and unthreatening. The implementation was of a generic laptop technology with wireless access to the existing intranet service. This allowed members of the organisation to access sources of information through an interface with which they were already familiar. It is important to note that this was not the study of the introduction of a totally new mobile technology, with a new interface, 
functionality or information sources: this case provides the story of the mobilisation of the familiar and of the pre-existing technology and information sources. It is also interesting in that this implementation was to support already mobile workers: the aim was not to create discontinuous change in working practices but to augment and enable to evolution of existing working practices. Its implementation was also in an organisation in which structural and cultural norms valued obedience and rule following: it could rationally expected that in this environment resistance to the technology would be limited.

As the trajectory of the implementation unfolded it became clear that the ROVER units enabled officers to re-order their actions and behaviours in new and unexpected ways. In the uniformed site this became the basis of the development high levels of ambiguity about ROVER leading finally to resistance and the rejection of the ROVER units. Where high levels of ambiguity are present the sense-making process around information technology becomes, as Weick (1995) notes, based on the enlargement of small cues, interacting to flesh out hunches and of interpreting and reinterpreting the past and driven by plausibility rather than accuracy. This also emphasises the relevance of the work of Henfridsson (2000) and Mantovani and Spagnolli (2001) on the importance of understanding the relationship between ambiguity created by the implementation of information technology and the sense-making process. In the Detective site, where there was less ambiguity about possible effects of the implementation on spatial, temporal and work orders, the systems was embraced and embedded into existing work practices. The paper clearly demonstrates how ambiguity, over time, influences the trajectory of the implementation process.

The primary contribution of this research is to provide a rich description of the situated activities of these mobile workers: a description which demonstrates the complexity of the social and organisational issues related to the mobilisation of information technology. This is novel not only in the perspective taken but also on the nature of the technology implemented. The technology implemented was the mobilization of the whole information environment (from e-mail to specific and specialised applications). Much of the earlier work on mobile technologies focuses on the application of specific and new mobile technologies which fit a particular task (Wiberg 2001).

\section{REFERENCES}

Adams, D. L., R. R. Nelson, et al. (1992). "Perceived usefulness, ease of use and usage of information technology: a replication." MIS Quarterly 16(2): 227-247. 
Agarwal, R. (2000). Individual acceptance of information technologies. Framing the domains of IT management: projecting the furture threough the past. R. W. Zmud. Cincinnati: OH, Pinnaflex Press: 85-104.

Barrett, M. I. (1999). "Challenges of EDI adoption for electronic trading in the

London Insurance Market." European Journal of Information Systems 8: 1-15.

Baruch, Y. and L. Lambert (2002). Organizational Anxiety: Applying psychological metaphor into organizational theory. The 4th International Conference on Organizational Discourse:

Word-views, Work-views and World Views.

Castells, M. (1996). The Rise of the Network Society. Malden, MA, Blackwell.

Ciborra, C. U. (1999). "Notes on Improvisation and Time." Organizations. Accounting, Management and Information Technologies 9: 77-94.

Ciborra, U. C. and G. F. Lanzara (1994). "Formative contexts and information technology: understanding the dynamics of innovation in organsiations." Accounting, Management and Information Technologies 4(61-86).

Dahlbom, B. and F. Ljungberg (1998). "Mobile Informatics." Scandinavian Journal of Information Systems 10(1/2): 227-234.

Davidson, E., J (2002). "Technology frames and framing: a socio-cognitive investigation of requirments determination." MIS Quarterly 26(4): 329-358.

DeSanctis, G. and P. Monge (1999). "Introduction to the special issue: Communication processes for virtual organizations." Organization Science 10(6): 693-703.

Fineman, S. (1996). Emotion and organising. Handbook of organization studies. S. R. Clegg, C. Hardy and W. R. Nord. London, Sage: 543-565.

Frissen, V. A. J. (2000). "ICTs in the rush hour of life." Information Society 16: 65-75.

Gallivan, M. J. (2001). "Organizational Adoption and assimilation of complex technological innovations: development and application of a new framework." The DATA BASE for Advances in Information Systems 32(3): 51-85.

Glaser, B. and A. Strauss (1968). Time for dying. Chicago, Aldine.

Harris, S. G. (1994). "Organizational culture and individual sensemaking: A schema based perspective." Organizational Science 5(3): 309-321.

Healey, P. G. T. and A. J. Reeves (2001). Mobility and Participatory Status. Interventions in the social, cultural and interactional analysis of mobility, ubiquity and information \& communication technology, Cumberland lodge, windsor, uk.

Henfridsson, O. (2000). "Ambiguity in IT adaptation: making sense of First Class in a social work setting." Information Systems Journal 10(2): 87-104.

Hodgkinson, G. P. (2002). "Comparing Managers' Mental Models of Competition: Why Selfreport Measures of

Belief Similarity Won't Do." Organization Studies 23(1): 63-72.

Hodgkinson, G. P. and P. R. Sparrow (2002). The competent organisation. Buckingham: UK, Open University Press.

Jenkins, M. and S. Floyd (2001). "Trajectories in the Evolution of Technology: A Multi-level Study of Competition in Formula 1 Racing." Organization Studies 22(6): 945-969.

Kakihara, M. and C. Sørensen (2001). Mobility Reconsidered: Topological Aspects of Interaction. IRIS'24, Ulvik, Norway, University of Bergen.

Kakihara, M. and C. Sørensen (2002). Mobility: An Extended Perspective. Thirty-Fifth Hawaii International Conference on System Sciences (HICSS-35), Big Island, Hawaii., IEEE.

Karahanna, E., D. W. Straub, et al. (1999). "Information technology adoption accross time: a cross-sectional comparison of pre-adoption and post-adoption beliefs." Managment Information Systems Quarterly 23(2): 183-213. 
Lee, H. (1999). "Time and information technology: Monochronicity, polychronicity and temporal symmetry." European Journal of Information Systems 8(1): 16-26.

Lee, H. and J. Liebenau (2000). "Temporal effects of information systems on business processes: focusing on the dimensions of temporality." Accounting, Management and Information Technologies 10(3).

Lyytinen, K. and Y. Yoo (2002). "Research Commentary: The Next Wave of Nomadic Computing." Information Systems Research 13(4): 377-388.

Mantovani, G. and A. Spagnolli (2001). "Legitimating technologies: ambiguity as a premise for negotation in a networked institution." Information Technology \& People 14(3): 301 320.

McCaskey, M. B. (1982). The executive challenge: managing change and ambiguity. Marshfield: MA, Pitman.

Nandhakumar, J. (2001). "Managing time in a software factory: termporal and spatial organisations of IS development activities."

Nulden, U. and J. Lundin (2002). Introduction to the Mobile Informatics Mini-Track. ThirtyFifth Hawaii International Conference on System Sciences (HICSS-35), Big Island, Hawaii., IEEE.

O'Dowd, D. J. (1998). Beating Crime: HMIC Thematic Inspection Report 1998. http://www.homeoffice.gov.uk/hmic/beatcrim.htm, Her Majesty's Inspectorate of Constabulary.

Okoli, C., B. Ives, et al. (2002). The Mobile Conference Information System Unleashing Academic Conferences with Wireless Mobile Computing. 35th Hawaii International Conference on System Sciences - 2002, Big Island, Hawaii.

Orlikowski, W., J and D. C. Gash (1994). "Technological frames: making sense of information technology in organizations." ACM Transactions on Information Systems 12: 174-207.

Orlikowski, W. J. and J. Yates (2002). "It's About Time: Temporal Structuring in Organizations." Organization Science 13(6).

Povey, K. (2001). Open all hours: A thematic inspection report on the role of police visibility and accessibility in public reassurance. London, Her Majesty's Inspector of Constabulary: 1-191.

Siau, K., L. Ee-Peng, et al. (2001). "Mobile Commerce: Promises, Challenges, and Research Agenda." Journal of Database Management.

Strauss, A. (1993). Continual permutations of action. New York, Aldine De Gruyter.

Tan, F. B. and M. G. Hunter (2002). "THE REPERTORY GRID TECHNIQUE: A METHOD FOR THE STUDY OF COGNITION IN INFORMATION SYSTEMS." MIS Quarterly 26(1): 39-57.

Thompson, J. B. (1990). Ideology and modern culture: critical social theory in the era of mass communication. Cambridge, Polity Press.

Thompson, J. B. (1995). The media and modernity: a social theory of the media. Cambridge., Polity Press.

Venkatesh, V. (2000). "Determinants of Perceived Ease of Use:Integrating Control, Intrinsic Motivation,

and Emotion into the Technology Acceptance Model." Information Systems Research 11(4): 342-365.

Walsh, J. P. (1995). "Managerial and organizational cognition: notes from a trip down memory lane." Organization Science 6(3): 280-321.

Weick, K. (1995). Sensemaking in organisations. London, Sage. 
Wiberg, M. (2001). Collaboration on the move: An empirical study of mobile work at Telia Nära. 24th Information Systems Research Seminar in Scandinavia (IRIS 24), Ulvik in Hardanger, Norway. 